\title{
Dietary Habits and Abdominal Pain-related Functional Gastrointestinal Disorders: A School-based, Cross-sectional Analysis in Greek Children and Adolescents
}

\author{
Giorgos Chouliaras, ${ }^{*}$ Christina Kondyli, Ilias Bouzios, Nick Spyropoulos, George P Chrousos, and Eleftheria Roma-Giannikou \\ First Department of Pediatrics, University of Athens, “Aghia Sophia” Children's Hospital, Athens, Greece
}

\section{Background/Aims}

The abdominal pain-related functional gastrointestinal disorders (AP-FGIDs) affect a significant proportion of the pediatric population and consist 1 of the most frequent causes for seeking medical advice. In this study, we aimed to assess the relation of dietary habits with the likelihood of AP-FGIDs.

\section{Methods}

This was a school-based, cross-sectional study approved by the Greek Government authorities, after obtaining informed consent by the legal representatives of the children. Diagnoses of AP-FGIDs were based on the Greek official translation of the Rome III questionnaire. Demographic, socioeconomic and dietary data were collected through self-reporting or parent-reporting questionnaires. Associations between the probability of AP-FGIDs and dietary practices were assessed after adjusting for known confounders through a multiple logistic regression analysis.

\section{Results}

A total of 1365 children (147 AP-FGIDs and 1218 controls, 52.4\% females, mean age: $12.8 \pm 2.8$ years) were included. Multiple regression analysis identified the following statistically significant confounders: victimization, the presence of a person with a severe health problem at home, female sex, engaging in limited physical exercise, and living in a single adult family. Subsequently, logistic regression, adjusted for the abovementioned confounders, showed that reduced fish and increased junk food consumption were related to a higher likelihood of AP-FGIDs.

\section{Conclusions}

Children with AP-FGIDs report excessive junk-food and reduced fish intake compared to controls. Further studies are needed in order to clarify the nature of this observation.

(J Neurogastroenterol Motil 2019;25:113-122)

Key Words

Children; Feeding behavior; Gastrointestinal diseases

Received: September 18, 2017 Revised: April 3, 2018 Accepted: September 28, 2018

(.) This is an Open Access article distributed under the terms of the Creative Commons Attribution Non-Commercial License (http://creativecommons. org/licenses/by-nc/4.0) which permits unrestricted non-commercial use, distribution, and reproduction in any medium, provided the original work is properly cited.

*Correspondence: Giorgos Chouliaras, PhD

First Department of Pediatrics, University of Athens, "Aghia Sophia" Children's Hospital, Thivon and Papadiamantopoulou str, 11527 Athens, Greece

Tel: +30-2107467945, Fax: +30-6972832283, E-mail: georgehouliaras@msn.com 


\section{Introduction}

The functional gastrointestinal disorders (FGIDs) affect a high proportion of school-aged children worldwide, approximately 1 out of 4 children in the general population. ${ }^{1,2}$ According to a recent meta-analysis, the subset of abdominal pain-related FGIDs (APFGIDs) has an estimated prevalence of $13.5 \% .^{3}$ The chronic and disabling nature of the symptoms is a major concern for pediatric public health ${ }^{4}$ in terms of financial burden and reduced quality of life for the patients and their families. ${ }^{5,6}$

The impact of FGIDs is significant in every aspect of medical practice, underlying the necessity for effective, therapeutic or preventing interventions. The lack of such options reflects the unknown aetiology of these disorders. Promising theories include stress-mediated somatization, brain-gut interactions, intestinal hypersensitivity, altered intestinal microbiota ${ }^{7}$ and life-style/socioeconomic factors. ${ }^{1,8}$ The role of diet remains unclear. ${ }^{9}$ Some data support a link between dietary habits and exacerbation of symptoms. ${ }^{10,11}$ Studies on specific dietary parameters have mainly focused on fiber and carbohydrates ${ }^{12}$ and have yielded inconclusive results. Therefore, the role of diet as a triggering, or even causal, factor in pediatric populations with AP-FGIDs remains to be elucidated. In addition, previous studies on food habits and FGIDs. ${ }^{10,11}$ in children have focused mainly on food-induced exacerbation of symptoms, included small, clinical samples and utilized univariate analyses. Therefore data and associations representative of feeding behaviour in children with AP-FGIDs are derived from small, clinical samples, are symptom-driven, based on univariate analyses and mainly focused on selected food groups.

In this study we sought to describe the dietary habits in a large, non-clinical sample of Greek children with AP-FGIDs and compare them with those of the general population, through a multivariate analysis. The study focused on identifying differences on multiple specific dietary factors such as fiber, dairy, simple carbohydrates, meat, junk food and fish.

\section{Materials and Methods}

The cross-sectional, school-based study presented in this paper was carried out by the 1st Department of Pediatrics of the National and Kapodistrian University of Athens, Greece, between January 2014 and June 2014. The study was approved by the Ethics Committee of the Ministry of Education (approval reference Id: 305/2505-2012). Written informed consent was obtained from the parents or legal guardians of all children who took part in the study. According to the instructions given by the Ministry of Education an assent form filled by older children was not required and therefore was not obtained in our sample.

The methodology is discussed in detail in a previous paper. ${ }^{1}$ In brief, children from public and private schools (primary schools: 6-11 year olds, gymnasiums: 12-14 year olds and lyceums: 15-17 year olds) were invited to participate via a letter sent to their parents. Consenting responders were included in the study. Data (FGID symptoms, demographic/socioeconomic information, and dietary information) were obtained through questionnaires filled by the parents of children younger than 12 years, or directly by older children. Collected information included:

\section{Rome III Questionnaire}

The official Greek translation of the Rome III questionnaire (QPGS-RIII) was utilized and all children were classified as having (or not) the following AP-FGIDs: functional dyspepsia, irritable bowel syndrome (IBS), abdominal migraine, functional abdominal pain (FAP) and functional abdominal pain syndrome (FAPS). ${ }^{13}$ Two groups were formed: Children with AP-FGIDs and controls. Children fulfilling criteria for other FGIDs were excluded from the control group.

\section{Demographic/Socioeconomic Information}

Geographic data: urban/rural, mainland/islands, main urban centers/elsewhere; sex; age; paternal and maternal educational level were recorded as 4-level scales: primary school $=0$, gymnasium $=1$, lyceum $=2$, and higher degree $=3$. For simplicity, the 2 variables were combined (by summing) to form a new variable, the combined parental educational level which was treated as a continuous parameter; family's economic status: poor, average, good (based on the personal perception of the participants and not on a pre-defined economic scale); number of adults at home; number of children at home; family size: 2-3 persons, 4-5 persons, and 6-8 persons; parental presence at home: single-parent families or both parents present; television exposure: $\leq 1$ hour per day, 1-3 hours per day, and $>3$ hours per day; victim of bullying at school: yes/no; and presence of a person with a severe health problem at home: yes/ no; level of physical exercise: none, 1-3 days per week, and 4-7 days per week.

\section{Dietary Information}

The following dietary parameters were of interest in the present analysis: dairy, meat, fish, fiber, junk food, and non-lactose-simple 
carbohydrates. The questionnaire used in this survey is a modified version of a frequency-based questionnaire of dietary habits published previously. ${ }^{14}$ In detail, most questions from the section $\mathrm{B}$ from the respective questionnaire were utilized, with the exception of pasta/rice/bread/potatoes, eggs, coffee and alcoholic drinks. The basis of exclusion was that these particular foods were not included in the objectives of the present analysis. For ham, salami and sausages, the respective question was omitted because these types of food are usually included in the fast food or savoury snacks or are in regular meals and were included in the junk food group. In the final form of the questionnaire savoury snacks included chips, crisps, pastries, salty biscuits, meat included red or white meat and junkfood included pizza, burgers, hot-dog, Greek pita-bread with kebab (and other type of meat products), sandwiches from kiosks and take-away shops ("submarine" type). The questions were modified in terms of the available answers (continuous response rather than ordinal). The time span remained 1 week. Detailed description of the questionnaire and grouping of variables is provided in Table 1. For the analysis, data (servings per week/occasions of consumption per week) for all foods included in the same group were summed. In all occasions, frequencies and not actual quantities were assessed. To assess whether the questionnaire provided accurate information on the frequencies of food consumption, a small validation study was performed, including a total of 50 children (25 aged 12-18 years and 25 aged 6-12 years). Initially, the participants (if aged $>$ 12 years) or their parents (if aged $<12$ years) completed the questionnaire in a retrospective manner, as was done in the main analy- sis. Subsequently, they were asked to fill in the questionnaire on a prospective basis, for 2 consecutive weeks. The degree of agreement between the retrospective and prospective data was assessed by the intra-class correlation coefficient. For all food groups, the values of intra-class correlation coefficient were between 0.80-0.90, indicating good agreement. Therefore, the data collected by the retrospective administration and completion of the questionnaire were considered valid, in terms of the recorded frequencies. No age-related differences were observed in terms of the validity of the adapted questionnaire.

\section{Statistical Methods}

Continuous variables are presented using the mean $\pm \mathrm{SD}$, median (interquartile rage $[\mathrm{IQR}]$ ) whereas categorical variables are described using absolute $(\mathrm{n})$ and relative $(\%)$ frequencies. Comparisons of continuous variables between groups were performed using the Student's $t$ test or the Mann-Whitney $U$ test in the case of skewed distributions. The relations between categorical variables were evaluated using Fisher's exact test. All the analyses were performed overall, as well as according to age ( $\geq 12$ years and $<12$ years) which corresponds to self- or parent-administered questionnaires. In a small number of participants, some fields in the questionnaires were left blank (missing values). Therefore in some variables the summed total might be lower than the total number of participants.

In a previous study in the same population, ${ }^{1}$ we showed that socioeconomic and demographic background is related to the likeli-

Table 1. Food-frequency Questionnaire and Grouping of Data

\begin{tabular}{ll}
\hline \multicolumn{1}{c}{ Question } & \multicolumn{1}{c}{ Group } \\
\hline Occasions of fruit consumption per week (in any form) & Fiber, non-lactose simple carbohydrates \\
Servings of fresh juice per week & Non-lactose simple carbohydrates \\
Fizzy drinks (cups or cans) per week & Non-lactose simple carbohydrates \\
Occasions of sweets consumption per week & Non-lactose simple carbohydrates \\
Occasions of savoury ${ }^{a}$ snacks consumption per week & Junk food \\
Servings of milk per week & Dairy \\
Occasions of cheese consumption per week & Dairy \\
Servings of yoghurt per week & Dairy \\
Out of the 14 meals per week how many include vegetables/legumes & Fiber \\
Out of the 14 meals per week how many include salad & Fiber \\
Out of the 14 meals per week how many include meat ${ }^{b}$ & Meat \\
Out of the 14 meals per week how many include fish & Fish \\
Out of the 14 meals per week how many include fast food ${ }^{c}$, exclusively $^{c}$ & Junk food \\
In how many out of the 14 meals per week, additional fast food is consumed after the meal & Junk food
\end{tabular}

${ }^{a}$ Chips, crisps, pastries, and salty biscuits.

${ }^{b}$ White or red meat.

"Pizza, burgers, hot-dog, Greek pita-bread with kebab (and other type of meat products), sandwiches from kiosks, and take-away shops ("submarine" type). 
hood of any-FGID. As a first step in the present analysis, we implemented a stepwise logistic regression approach incorporating all socioeconomic and demographic variables to identify probable confounders. During the second phase of the analysis another stepwise logistic regression model was applied where correlations of dietary habits to the likelihood of AP-FGIDs were assessed. The set of statistically significant parameters derived from the first model was forced in the final equation (ie, it was not subjected to the elimination process of the stepwise algorithm). For the stepwise regression analyses, variables were excluded or retained using $P<0.05$ as the cut-off point. Results are reported as odds ratios (OR) and 95\% confidence intervals (95\% CI). The analysis was carried out with Stata 11.0 MP (StataCorp, College Station, Texas, USA).

\section{Results}

\section{Descriptive Statistics}

A total of 1365 children (147 AP-FGIDs and 1218 controls, $52.4 \%$ females, mean age: $12.8 \pm 2.8$ years) were included in the analysis. One type of AP-FGID was diagnosed in 130 children, 2 types of AP-FGIDs in 16 (14 with abdominal migraine and IBS and 2 with abdominal migraine and functional dyspepsia) and 3 types of AP-FGIDs in 1 child (abdominal migraine, IBS, and functional dyspepsia). Abdominal migraine was diagnosed in 89 participants, functional dyspepsia in 7, IBS in 48, FAP in 14 and FAPS in 7.

Descriptive statistics on socioeconomic and demographic information and differences between children with AP-FGIDs and controls are presented in Tables 2 and 3. Comparisons of dietary parameters between study groups are shown in Table 4 . In the entire study sample children with AP-FGIDs reported more frequent consumption of junk foods as well as lower consumption of fish compared to controls. In children younger than 12 years no statistically significant differences were observed. In older children, the analysis yielded results similar to the pooled analysis for non-lactose simple carbohydrates, fiber, fish, meat, and junk food, whereas APFGIDs patients reported lower frequency of dairy consumption compared to controls.

\section{Initial Multivariate Analysis for the Identification of Probable Confounding Parameters}

Results of initial multivariate logistic regression analysis, including demographic and socioeconomic factors, showed that being

Table 2. Demographic Factors in the Study Population and Association With Abdominal Pain-related Functional Gastrointestinal Disorders

\begin{tabular}{|c|c|c|c|c|}
\hline Demographic characteristics & Total $(\mathrm{N}=1365)$ & AP-FGIDs $(n=147)$ & Controls $(\mathrm{n}=1218)$ & $P$-value ${ }^{\mathrm{a}}$ \\
\hline Group & & & & 0.990 \\
\hline Primary school (6-12 yr) & $363(26.6)$ & $39(26.5)$ & $324(26.6)$ & \\
\hline High school (gymnasium and lyceum, 12-18 yr) & $1002(73.4)$ & $108(73.5)$ & $894(73.4)$ & \\
\hline Sex & & & & $<0.001$ \\
\hline Females & $715(52.4)$ & $104(70.7)$ & $611(50.2)$ & \\
\hline Males & $649(47.6)$ & $43(29.3)$ & $606(49.8)$ & \\
\hline Main urban & & & & 0.725 \\
\hline Athens \& Thessaloniki & $778(57.0)$ & $86(58.5)$ & $692(56.9)$ & \\
\hline Elsewhere & $586(43.0)$ & $61(41.5)$ & $525(43.2)$ & \\
\hline Urban vs rural & & & & 0.849 \\
\hline Rural & $412(30.2)$ & $43(29.3)$ & $369(30.3)$ & \\
\hline Urban & $952(69.8)$ & $104(70.7)$ & $848(69.7)$ & \\
\hline Islands vs Mainland Greece & & & & 0.549 \\
\hline Mainland Greece & $1149(84.2)$ & $127(86.4)$ & $1022(84.0)$ & \\
\hline Islands & $215(15.8)$ & $20(13.6)$ & $195(16.0)$ & \\
\hline Variable & Total & AP-FGID & Controls & $P$-value ${ }^{\mathrm{b}}$ \\
\hline Age (yr) & $\begin{array}{c}12.8 \pm 2.8 \\
13.3(11.8,14.5)\end{array}$ & $\begin{array}{c}13.3 \pm 3.0 \\
13.8(11.9,15.5)\end{array}$ & $\begin{array}{c}12.8 \pm 2.8 \\
13.2(11.8,14.5)\end{array}$ & 0.045 \\
\hline
\end{tabular}

${ }^{a}$ Fisher's exact test.

'Student's $t$ test.

AP-FGIDs, abdominal pain-related functional gastrointestinal disorders.

Data are presented as $\mathrm{n}(\%)$ or mean $\pm \mathrm{SD}$, median (interquartile range). 
Table 3. Socioeconomic Factors in the Study Population and Association With Abdominal Pain-related Functional Gastrointestinal Disorders

\begin{tabular}{|c|c|c|c|c|}
\hline Socioeconomic characteristics & Total $(\mathrm{N}=1365)$ & AP-FGIDs $(\mathrm{n}=147)$ & Controls $(n=1218)$ & $P$-value ${ }^{a}$ \\
\hline Paternal educational level & & & & 0.547 \\
\hline Primary school & $61(4.5)$ & $9(6.1)$ & $52(4.3)$ & \\
\hline Gymnasium & $188(13.8)$ & $16(10.9)$ & $172(14.1)$ & \\
\hline Lyceum & $532(39.0)$ & $59(40.1)$ & $473(38.9)$ & \\
\hline University & $583(42.7)$ & $63(42.9)$ & $520(42.7)$ & \\
\hline Maternal educational level & & & & 0.580 \\
\hline Primary school & $61(4.5)$ & $9(6.1)$ & $52(4.3)$ & \\
\hline Gymnasium & $95(7.0)$ & $8(5.4)$ & $87(7.1)$ & \\
\hline Lyceum & $556(40.7)$ & $63(42.9)$ & $493(40.5)$ & \\
\hline University & $652(47.8)$ & $67(45.6)$ & $585(48.1)$ & \\
\hline Family's economic status & & & & 0.147 \\
\hline Good & $518(38.0)$ & $48(32.7)$ & $470(38.6)$ & \\
\hline Average & $766(56.1)$ & $86(58.5)$ & $680(55.8)$ & \\
\hline Poor & $81(5.9)$ & $13(8.8)$ & $68(5.6)$ & \\
\hline Number of adults at home & & & & 0.003 \\
\hline 1 & $95(7.0)$ & $18(12.2)$ & $77(6.3)$ & \\
\hline 2 & $1111(81.4)$ & $104(70.8)$ & $1007(82.7)$ & \\
\hline 3 & $107(7.8)$ & $15(10.2)$ & $92(7.6)$ & \\
\hline 4 & $52(3.8)$ & $10(6.8)$ & $42(3.4)$ & \\
\hline Family size (people at home) & & & & 0.267 \\
\hline $2-3$ & $231(16.9)$ & $28(19.0)$ & $203(16.7)$ & \\
\hline $4-5$ & $980(71.9)$ & $98(66.7)$ & $882(72.5)$ & \\
\hline $6-8$ & $153(11.2)$ & $21(14.3)$ & $132(10.9)$ & \\
\hline Parental presence at home & & & & 0.042 \\
\hline Single parent & $119(8.7)$ & $20(13.6)$ & $99(8.1)$ & \\
\hline Both parents & $1246(91.3)$ & $127(86.4)$ & $1119(91.9)$ & \\
\hline Days of physical exercise per week & & & & 0.003 \\
\hline None & $229(16.8)$ & $35(23.8)$ & $194(15.9)$ & \\
\hline $1-3$ & $754(55.3)$ & $86(58.5)$ & $668(54.9)$ & \\
\hline $4-7$ & $381(27.9)$ & $26(17.7)$ & $355(29.2)$ & \\
\hline Television exposure (hours per day) & & & & 0.417 \\
\hline 1 hour or less & $517(37.9)$ & $52(35.4)$ & $465(38.2)$ & \\
\hline $1-3$ hours & $782(57.3)$ & $85(57.8)$ & $697(57.2)$ & \\
\hline More than 3 hours & $66(4.8)$ & $10(6.8)$ & $56(4.6)$ & \\
\hline Victim of "bullying" at school & & & & 0.002 \\
\hline No & $1231(90.2)$ & $121(82.3)$ & $1110(91.1)$ & \\
\hline Yes & $134(9.8)$ & $26(17.7)$ & $108(8.9)$ & \\
\hline Person at home with a severe health problem & & & & 0.002 \\
\hline No & $1266(92.7)$ & $126(85.7)$ & $1140(93.6)$ & \\
\hline Yes & $99(7.3)$ & $21(14.3)$ & $78(6.4)$ & \\
\hline Variable & $\begin{array}{c}\text { Total } \\
(\mathrm{N}=1365)\end{array}$ & $\begin{array}{c}\text { AP-FGIDs } \\
(\mathrm{n}=147)\end{array}$ & $\begin{array}{c}\text { Controls } \\
(\mathrm{n}=1218)\end{array}$ & $P$-value ${ }^{\mathrm{b}}$ \\
\hline Number of children at home & $2.2 \pm 0.9,2(2,2)$ & $2.3 \pm 1.0,2(2,3)$ & $2.2 \pm 0.9,2(2,2)$ & 0.168 \\
\hline Parental combined educational level $^{c}$ & $4.5 \pm 1.4,5(4,6)$ & $4.5 \pm 1.5,5(4,6)$ & $4.5 \pm 1.4,5(4,6)$ & 0.790 \\
\hline
\end{tabular}

${ }^{a}$ Fisher's exact test.

${ }^{\mathrm{b}}$ Mann-Whitney $U$ test.

'The combined parental educational level is a 0-6 scale resulting from summing the maternal and paternal educational level: primary school, 0 ; gymnasium, 1 ; lyceum, 2; and higher degree, 3.

AP-FGIDs, abdominal pain-related functional gastrointestinal disorders.

Data are presented as $\mathrm{n}(\%)$ or mean $\pm \mathrm{SD}$, median (interquartile range). 
Table 4. Distribution of Dietary Parameters in the Study Population and Comparison Between Children With Abdominal Pain-related Functional Gastrointestinal Disorders and Controls

\begin{tabular}{|c|c|c|c|c|}
\hline Dietary variables $^{a}$ & Total & AP-FGIDs & Controls & $P$-value \\
\hline \multicolumn{5}{|l|}{ Overall } \\
\hline Fiber & $18.3 \pm 9.1,17(11,24)$ & $17.9 \pm 9.3,17(11,24)$ & $18.4 \pm 9.1,17(11,24)$ & 0.426 \\
\hline Dairy & $16.5 \pm 7.6,16(11,21)$ & $15.7 \pm 7.8,16(11,20)$ & $16.5 \pm 7.5,16(11,21)$ & 0.341 \\
\hline Non-lactose-simple carbohydrates & $16.5 \pm 8.2,15(11,21)$ & $17.2 \pm 8.9,15(11,21)$ & $16.4 \pm 8.1,15(11,21)$ & 0.479 \\
\hline Junk food & $4.1 \pm 4.2,3(2,5)$ & $5.4 \pm 6.3,4(2,6)$ & $3.9 \pm 3.8,3(2,5)$ & 0.004 \\
\hline Meat & $5.0 \pm 2.7,4(3,7)$ & $5.2 \pm 3.0,5(3,7)$ & $5.0 \pm 2.7,4(3,6)$ & 0.521 \\
\hline Fish & $2.0 \pm 1.7,2(1,2)$ & $1.7 \pm 1.6,1(1,2)$ & $2.0 \pm 1.7,2(1,2)$ & 0.007 \\
\hline \multicolumn{5}{|l|}{ Children $<12$ years of age } \\
\hline Fiber & $17.6 \pm 8.1,17(11,23)$ & $16.5 \pm 7.8,15(11,20)$ & $17.8 \pm 8.1,17(11.5,23)$ & 0.269 \\
\hline Dairy & $18.6 \pm 5.8,19(15,23)$ & $19.6 \pm 4.9,19.5(16,23)$ & $18.5 \pm 5.9,19(14,23)$ & 0.220 \\
\hline Non-lactose-simple carbohydrates & $15.8 \pm 6.7,15(11,20)$ & $15.9 \pm 6.9,15(11,19)$ & $15.8 \pm 6.7,15(11,20)$ & 0.997 \\
\hline Junk food & $2.6 \pm 2.0,2(1,3)$ & $2.8 \pm 2.5,2(1,4)$ & $2.6 \pm 2.0,2(1,3)$ & 0.965 \\
\hline Meat & $4.9 \pm 2.4,4(3,6)$ & $4.8 \pm 2.6,3(3,7)$ & $4.9 \pm 2.3,4(3,6)$ & 0.730 \\
\hline Fish & $1.7 \pm 1.1,1(1,2)$ & $1.5 \pm 1.2,1(1,2)$ & $1.7 \pm 1.1,1(1,2)$ & 0.220 \\
\hline \multicolumn{5}{|l|}{ Children $\geq 12$ years of age } \\
\hline Fiber & $18.6 \pm 9.5,17.5(11,25)$ & $18.4 \pm 9.8,17(11.5,24)$ & $18.6 \pm 9.5,18(11,25)$ & 0.749 \\
\hline Dairy & $15.7 \pm 8.0,15(10,20)$ & $14.4 \pm 8.2,14(10,18.5)$ & $15.8 \pm 7.9,15(10,21)$ & 0.049 \\
\hline Non-lactose-simple carbohydrates & $16.8 \pm 8.7,14.5(11,22)$ & $17.6 \pm 9.5,15(11,21.5)$ & $16.7 \pm 8.6,14(11,22)$ & 0.412 \\
\hline Junk food & $4.6 \pm 4.6,4(2,6)$ & $6.4 \pm 6.9,4(3,7.5)$ & $4.4 \pm 4.2,3(2,6)$ & 0.001 \\
\hline Meat & $5.0 \pm 2.8,4(3,7)$ & $5.2 \pm 3.2,5(3,7)$ & $4.9 \pm 2.3,4(3,6)$ & 0.391 \\
\hline Fish & $2.1 \pm 1.8,2(1,3)$ & $1.8 \pm 1.8,1(1,2)$ & $2.1 \pm 1.8,2(1,3)$ & 0.016 \\
\hline
\end{tabular}

${ }^{2}$ Occasions or servings of consumption per week as defined in methodology. Numbers describe the sum of servings per week/occasions of consumption per week for all foods included in the same group.

${ }^{\mathrm{b}}$ Mann-Whitney $U$ test.

AP-FGIDs, abdominal pain-related functional gastrointestinal disorders.

Data are presented as mean $\pm \mathrm{SD}$, median (interquartile range).

the victim of bullying (OR, 2.0; 95\% CI, 1.2-3.3; $P=0.005)$, the presence of a person with a severe health problem at home $(\mathrm{OR}, 2.0$; $95 \% \mathrm{CI}, 1.1-3.4 ; P=0.017)$, being female (OR, 2.2; 95\% CI, 1.53.2; $P<0.001$ ), engaging in limited physical exercise (none vs $4-7$ days/week: OR, 1.9; 95\% CI, 1.1-3.3; $P=0.028)$, and living in a single adult family (single adult vs 2 adults at home: OR, 2.0; $95 \%$ CI, $1.1-3.5 ; P=0.018$ ) were positively related to the likelihood of AP-FGIDs and statistically significant.

\section{Regression Analysis of Dietary Habits on the Probability of Abdominal Pain-related Functional Gastrointestinal Disorders}

A second multivariate regression analysis was applied to estimate associations between dietary habits and the likelihood of APFGIDs. All significant parameters derived from the previous model were used to adjust for confounding effects. Of all dietary data, the frequency of fish and junk food consumption were significantly related to the probability of FGIDs and included in the final equa- tion. In detail, higher junk food consumption was associated with increased odds of AP-FGIDs (OR, 1.07; 95\% CI, 1.03-1.10; $P<$ $0.001)$, whereas higher fish consumption was related to lower odds of AP-FGIDs (OR, 0.87; 95\% CI, 0.77-0.98; $P=0.027$ ).

The second regression analysis was also performed independently after stratifying according to age (older or younger than 12 years). For older children the results were identical to those derived from the pooled data (junk food: OR, 1.07; 95\% CI, 1.03-1.11; $P<0.001$; fish: OR, 0.87; 95\% CI, 0.76-0.99; $P=0.042)$ whereas in younger children no significant relations were found. Nevertheless it should be mentioned that the direction of relations was similar to the entire population.

\section{Discussion}

The present analysis showed that specific dietary habits are more frequent in children with AP-FGIDs than controls, specifically increased junk food and reduced fish consumption. In multi- 
factorial disorders, such as the AP-FGIDs, researchers attempt to identify parameters that contribute to the pathogenesis of the conditions, rather than aiming at a single causative agent. The design of the present analysis had that precise orientation; therefore a multivariate analysis was used, acknowledging that several other factors must be taken into consideration irrespective of dietary habits.

To date research on AP-FGIDs and diet has focused mainly on fiber, lactose and other carbohydrates. Horvath and colleagues in a systematic review concluded that supplemental fiber does not affect AP-FGID. ${ }^{15}$ In relation to lactose intolerance, several studies in children have failed to provide evidence supportive of this hypothesis. ${ }^{16,17}$ A 2009 Cochrane metanalysis did not find convincing evidence that a lactose-free diet is beneficial for patients with FAP/IBS. ${ }^{18}$ In contrast to fiber and lactose, a significant effect on pain, as well as other gastrointestinal symptoms, has been identified for fructose. ${ }^{19-21}$ In the same context, attention has been drawn on diets of low fermentable oligosaccharides, disaccharides, monosaccharides, and polyols (FODMAP). In children, Chumpitazi et $\mathrm{al}^{22}$ provided evidence that FODMAP restriction could lead to alleviation of FAP, possibly via a microbiome modulation-mediated mechanism. Although carbohydrate malabsorption can induce pain-related gastrointestinal symptoms, the existing evidence do not strongly support avoidance of single carbohydrates in children with AP-FGIDs. ${ }^{12}$

In the setting of food habits, few studies have examined possible assocations with AP-FGIDs. In a small, clinical, not controlled study of 25 adolescents with diagnosed FGIDs, ${ }^{11}$ spicy foods were identified by both patients and parents as the most common food group perceived to cause symptom exacerbation. In a recent study, ${ }^{10}$ Reed-Knight and colleagues assessed 99 adolescents (48 IBS patients and 51controls) focusing on identifying differences in eating-associated symptoms. Dairy, carbohydrates, fruit, vegetables and fat were significantly more frequently related to gastrointestinal symptoms compared to controls. Patients consumed less total fat, less saturated fat and less lactose. Omega-3 lipids intake was, also, lower in IBS patients compared to controls, although the result was not statistically significant $(P=0.120)$. In our study, quantification of dietary composition was not available and therefore direct comparisons to the abovementioned results are not feasible. Nevertheless, some general remarks can be made. In our univariate analyses, in children older than 12 years, consumption of dairy was lower in AP-FGIDs children, a finding in accordance to the quantified analysis of lactose in the article by Reed-Knight et al. ${ }^{10}$ However, in the multivariate analysis in our population, the significance was lost. We found less frequent fish consumption which, in Greece, is the main dietary source of omega-3 lipids. Similarly, in the abovementioned study there was a trend for lower omega-3 intake in IBS children but failed to reach statistical significance. In contrast, the authors reported that saturated fat consumption was significantly reduced in IBS children, whereas in our population foods containing saturated fat (junk food) were more frequently consumed by AP-FGIDs patients. The reasons for the observed discrepancy, apart from the lack of quantification in our study, could be related to the nature of the sample. In contrast to the study by Reed-Knight and colleagues, where a clinical sample of IBS patients was evaluated, we included a school-based sample of AP-FGIDs children. Therefore, knowledge of the disease and subsequent physician-guided or self-guided modifications of the diet may have altered the results. The sample size was relatively small and omega-3 consumption might have reached statistical significance if more power was available. Last, the authors did not performed a multivariate analyses although the available data, including caloric intake, allowed such an approach.

We did not find a relation between AP-FGIDs and consumption of foods containing fiber, non-lactose-simple carbohydrates and dairy but they suggest an association to fish and fast food frequency consumption. In relation to fish intake, a plausible mechanism could be based on the high content of eicosapentanoic, docosahexanoic, and docosapentanoic acids in fish/fish oils. Other research groups have shown that patients with IBS exhibit disturbed lipid profile and reduced levels of polyunsaturated fatty acids. ${ }^{23}$ Kilkens et $\mathrm{al}^{24}$ linked the altered lipid profile to affective dysregulation, which is a common behavioral finding in patients with IBS. In autistic children, a similar association between lower docosahexanoic acid and gastrointestinal symptoms has also been postulated. ${ }^{25}$ The most relevant and supportive data, for this hypothesis, were recently published by Alfven and Strandvik. ${ }^{26}$ In their study, omega-3 and omega-6 fatty acids were evaluated in children with recurrent abdominal pain and controls. The authors concluded that an imbalance between proinflammatory "nociceptive" omega- 6 fatty acids and anti-inflammatory, "antinociceptive" omega-3 fatty acids might contribute, through a stress-related pathway, to abdominal pain.

Very few data on the relation of FGIDs and junk food consumption have been published. In an large $(\mathrm{n}=2034)$, schoolbased pediatric population from Taiwan junk food consumption was more frequent $(\mathrm{OR}=1.8)$ among patients with FGIDs. ${ }^{27}$ Junk food consumers are characterized by higher intake of saturated/trans fatty acids, preservatives, processed carbohydrates, and salt, as well as lack of fiber. ${ }^{28}$ In the multivariate analysis, simple carbohydrates and fiber intake were not statistically significant. In view of this, we speculate that the amount and type of lipids in the 
diet is the key to interpreting the association between junk food and AP-FGIDs. Some human data have linked the amount of fat intake to intestinal motility, ${ }^{29,30}$ whereas experimental studies in rats have demonstrated structural adaptation of the villi and enterocytes to high-fat diet. ${ }^{31}$ Another candidate to explain a possible association between junk food and FGID is the intestinal microbiota. Highfat diets, especially with significant amounts of saturated lipids, change the composition of the dominant bacterial populations, thus favoring an increase in the gram-negative to gram-positive ratio. ${ }^{32,33}$ The connection between gut microbiota and FGIDs has been the subject of intense research, both at the basic level, ${ }^{34,35}$ but also in the clinical context. This might explain the beneficial effect of certain probiotics. $^{36}$

Other substances found in excess in junk-food are salt and preservatives. Both are extremely difficult to be quantified and although both are considered harmful for human health, no data in relation to FGIDs exist.

Some indirect evidence may also be derived from the relation of obesity and FGIDs. The association between junk food and obesity is well established. ${ }^{37}$ Obese children have an almost 2-fold increase of risk compared to non-obese individuals for FGIDs. Due to the cross-sectional, observational design of all abovementioned studies their results may serve only as guidance for future research and not for firm conclusions on the interplay between diet, obesity and APFGIDs.

Taken together, the dietary parameters in the final regression model could reflect an imbalance in dietary fat, generated by a high intake of saturated fats and low intake of omega- 3 polyunsaturated fatty acids. This observation, although in agreement with experimental data, needs to be further tested in an appropriately designed framework, ideally clinical trials with dietary interventions and/ or supplementation with omega-3 fatty acids. It has to be noted that, in the final model, all initial confounders retained their significance and fish/junk food emerged as additional significant factors. Therefore, children with the particular socioeconomic profile and unhealthy dietary habits report the highest rates of AP-FGIDs in the study population.

The age-stratified analysis produced similar, to the overall, results for children older than 12 years, whereas no statistical differences were observed in younger children, although the direction of associations was similar. That could be attributed to the small sample size in the latter group (363 children with only 39 cases of AP-FGIDs) which provides low power for statistical purposes. Nevertheless, it could be as well an indirect indication of the suitability of the food frequency questionnaire in children younger than
12 years, taking into account that the original version was developed for adolescents.

The major strength of our study is the large sample, which provided the necessary power to assess multivariate models. Therefore, all the reported correlations between AP-FGIDs and dietary habits were adjusted for several confounding factors identified by the initial regression model.

The main drawback of our study is the cross-sectional design, which allows assessment of correlations but not causal inference. In addition, the dietary habits were evaluated via a self-report, semiquantitative questionnaire based on the frequency of consumption rather than the actual quantity of foods. Inter-observer variation (parent-reported vs child-reported) cannot be ruled out. No total caloric intake or specific nutritional sub-groups were recorded. A significant issue is related to the absence of information on gluten consumption. Gluten has been proposed as a causative factor for AP-FGIDs through the non-celiac gluten hypersensitivity. ${ }^{38}$ The nature of the questionnaire makes it impossible even to semiquantify gluten, therefrom the lack of such data. Several items of the original questionnaire were omitted as they were not included in the objectives of the analysis (eg, potatoes, rice, alcohol, and coffee). No question regarding adherence to a vegetarian diet was included and therefore no such analysis could be performed. Having said that, only 2 children recorded no meat/fish/junk food consumption, indicating that the prevalence of vegetarians in our sample would be very low prohibiting any statistical analysis. Finally, the original version of the questionnaire was assessed in adolescents and not in children younger than 12 years. Nevertheless, no differences between different age groups were observed in the small validation study that was performed prior to the distribution of the questionnaire

Summarizing our results, dietary excess in junk food and lack of fish consumption are significantly more frequent in children with AP-FGIDs, regardless of other factors associated with AP-FGIDs. Although some promising theories can be formulated on the basis of our findings, the nature of the relation remains to be elucidated in prospective interventional studies

\section{Financial support: None.}

\section{Conflicts of interest: None.}

Author contributions: Giorgos Chouliaras conceptualized and designed the study, performed all analyses, and wrote the manuscript; Christina Kondyli designed the dietary questionnaire and wrote the manuscript; Ilias Bouzios and Nick Spyropoulos 
supervised data collection and wrote the manuscript; and Eleftheria Roma-Giannikou and George P Chrousos designed the study, supervised the project, and edited the manuscript.

\section{References}

1. Bouzios I, Chouliaras G, Chrousos GP, Roma E, Gemou-Engesaeth V. Functional gastrointestinal disorders in Greek children based on Rome III criteria: identifying the child at risk. Neurogastroenterol Motil 2017;29.

2. Lewis ML, Palsson OS, Whitehead WE, van Tilburg MA. Prevalence of functional gastrointestinal disorders in children and adolescents. J Pediatr 2016;177:39-43, e33.

3. Korterink JJ, Diederen K, Benninga MA, Tabbers MM. Epidemiology of pediatric functional abdominal pain disorders: a meta-analysis. PLoS One 2015;10:e126982.

4. Park R, Mikami S, LeClair J, et al. Inpatient burden of childhood functional GI disorders in the USA: an analysis of national trends in the USA from 1997 to 2009. Neurogastroenterol Motil 2015;27:684-692.

5. Dhroove G, Chogle A, Saps M. A million-dollar work-up for abdominal pain: is it worth it? J Pediatr Gastroenterol Nutr 2010;51:579-583.

6. Drossman DA, Li Z, Andruzzi E, et al. U.S. householder survey of functional gastrointestinal disorders. Prevalence, sociodemography, and health impact. Dig Dis Sci 1993;38:1569-1580.

7. Korterink J, Devanarayana NM, Rajindrajith S, Vlieger A, Benninga MA. Childhood functional abdominal pain: mechanisms and management. Nat Rev Gastroenterol Hepatol 2015;12:159-171.

8. Tanaka Y, Kanazawa M, Fukudo S, Drossman DA. Biopsychosocial model of irritable bowel syndrome. J Neurogastroenterol Motil 2011;17:131-139.

9. van Tilburg MA, Felix CT. Diet and functional abdominal pain in children and adolescents. J Pediatr Gastroenterol Nutr 2013;57:141-148.

10. Reed-Knight B, Squires M, Chitkara DK, van Tilburg MA. Adolescents with irritable bowel syndrome report increased eating-associated symptoms, changes in dietary composition, and altered eating behaviors: a pilot comparison study to healthy adolescents. Neurogastroenterol Motil 2016;28:1915-1920.

11. Carlson MJ, Moore CE, Tsai CM, Shulman RJ, Chumpitazi BP. Child and parent perceived food-induced gastrointestinal symptoms and quality of life in children with functional gastrointestinal disorders. J Acad Nutr Diet 2014;114:403-413.

12. Chumpitazi BP, Shulman RJ. Dietary carbohydrates and childhood functional abdominal pain. Ann Nutr Metab 2016;68(suppl 1):8-17.

13. Rasquin A, Di Lorenzo C, Forbes D, et al. Childhood functional gastrointestinal disorders: child/adolescent. Gastroenterology 2006;130:15271537.

14. Turconi G, Celsa M, Rezzani C, Bïno G, Sartirana MA, Roggi C. Reliability of a dietary questionnaire on food habits, eating behaviour and nutritional knowledge of adolescents. Eur J Clin Nutr 2003;57:753-763.

15. Horvath A, Dziechciarz P, Szajewska H. Systematic review of randomized controlled trials: fiber supplements for abdominal pain-related functional gastrointestinal disorders in childhood. Ann Nutr Metab 2012;61:95-101.

16. Lebenthal E, Rossi TM, Nord KS, Branski D. Recurrent abdominal pain and lactose absorption in children. Pediatrics 1981;67:828-832.

17. Dearlove J, Dearlove B, Pearl K, Primavesi R. Dietary lactose and the child with abdominal pain. Br Med J (Clin Res Ed) 1983;286:1936.

18. Huertas-Ceballos AA, Logan S, Bennett C, Macarthur C. Dietary interventions for recurrent abdominal pain (RAP) and irritable bowel syndrome (IBS) in childhood. Cochrane Database Syst Rev 2009:CD003019.

19. Gomara RE, Halata MS, Newman LJ, et al. Fructose intolerance in children presenting with abdominal pain. J Pediatr Gastroenterol Nutr 2008;47:303-308.

20. Escobar MA Jr, Lustig D, Pflugeisen BM, et al. Fructose intolerance/ malabsorption and recurrent abdominal pain in children. J Pediatr Gastroenterol Nutr 2014;58:498-501.

21. Wintermeyer P, Baur M, Pilic D, Schmidt-Choudhury A, Zilbauer M, Wirth S. Fructose malabsorption in children with recurrent abdominal pain: positive effects of dietary treatment. Klin Padiatr 2012;224:17-21.

22. Chumpitazi BP, Cope JL, Hollister EB, et al. Randomised clinical trial: gut microbiome biomarkers are associated with clinical response to a low FODMAP diet in children with the irritable bowel syndrome. Aliment Pharmacol Ther 2015;42:418-427.

23. Solakivi T, Kaukinen K, Kunnas T, Lehtimäki T, Mäki M, Nikkari ST. Serum fatty acid profile in subjects with irritable bowel syndrome. Scand J Gastroenterol 2011;46:299-303.

24. Kilkens TO, Honig A, Maes M, Lousberg R, Brummer RJ. Fatty acid profile and affective dysregulation in irritable bowel syndrome. Lipids 2004;39:425-431.

25. Mostafa GA, Al-Ayadhi LY. Reduced levels of plasma polyunsaturated fatty acids and serum carnitine in autistic children: relation to gastrointestinal manifestations. Behav Brain Funct 2015;11:4.

26. Alfven G, Strandvik B. Antinociceptive fatty acid patterns differ in children with psychosomatic recurrent abdominal pain and healthy controls. Acta Paediatr 2016;105:684-688.

27. Shau JP, Chen PH, Chan CF, et al. Fast foods--are they a risk factor for functional gastrointestinal disorders? Asia Pac J Clin Nutr 2016;25:393401.

28. Bowman SA, Gortmaker SL, Ebbeling CB, Pereira MA, Ludwig DS. Effects of fast-food consumption on energy intake and diet quality among children in a national household survey. Pediatrics 2004;113(1 Pt 1):112118.

29. Clegg ME, McKenna P, McClean C, et al. Gastrointestinal transit, postprandial lipaemia and satiety following 3 days high-fat diet in men. Eur J Clin Nutr 2011;65:240-246.

30. Boyd KA, O'Donovan DG, Doran S, et al. High-fat diet effects on gut motility, hormone, and appetite responses to duodenal lipid in healthy men. Am J Physiol Gastrointest Liver Physiol 2003;284:G188-G196.

31. Thomson AB, Keelan M, Wild GE. Nutrients and intestinal adaptation. Clin Invest Med 1996;19:331-345.

32. Doré J, Blottiére $\mathrm{H}$. The influence of diet on the gut microbiota and its consequences for health. Curr Opin Biotechnol 2015;32:195-199. 
33. Cani PD, Everard A. Talking microbes: when gut bacteria interact with diet and host organs. Mol Nutr Food Res 2016;60:58-66.

34. Rigsbee L, Agans R, Shankar V, et al. Quantitative profiling of gut microbiota of children with diarrhea-predominant irritable bowel syndrome. Am J Gastroenterol 2012;107:1740-1751.

35. Saulnier DM, Riehle K, Mistretta TA, et al. Gastrointestinal microbiome signatures of pediatric patients with irritable bowel syndrome. Gastroenterology 2011;141:1782-1791.
36. Rutten JM, Korterink JJ, Venmans LM, Benninga MA, Tabbers MM. Nonpharmacologic treatment of functional abdominal pain disorders: a systematic review. Pediatrics 2015;135:522-535.

37. Rajindrajith S, Devanarayana NM, Benninga MA. Obesity and functional gastrointestinal diseases in children. J Neurogastroenterol Motil 2014;20:414-416.

38. Fasano A, Sapone A, Zevallos V, Schuppan D. Nonceliac gluten sensitivity. Gastroenterology 2015;148:1195-1204. 